\title{
Belt Aligning Revisited
}

\author{
Vadim Yurchenko ${ }^{1 *}$ \\ ${ }^{1}$ T.F. Gorbachev Kuzbass State Technical University, 650000, 28 Vesennyaya St., Kemerovo, Russia
}

\begin{abstract}
The misalignment causes the greatest damage to the conveyor belt. As a result of the interaction of the moving belt with the stationary parts of the conveyor, the sides of the belt wear intensively. This results in reducing the life of the belt. The reasons for this phenomenon are well investigated, but the difficulty lies in the fact that they all act simultaneously. The belt misalignment prevention can be carried out in two ways: by minimizing the effect of causes and by aligning the belt. The construction of aligning devices and errors encountered in practice are considered in this paper. Self-aligning roller supports rotational in plan view are recommended as a means of combating the belt misalignment.
\end{abstract}

\section{Introduction}

The misalignment of conveyor belts contributes to their active wear. According to the place where the causes of wear are concentrated, the conveyor belts are represented by the following elements: the traction core, the conveyor-belt edge surface, the carrying side cover and the bottom cover. The greatest influence on the strength properties of the belts and their serviceability is provided by the causes associated with the wear of the conveyorbelt edge surface (abrasion and breaking). The complexity of the problem lies in the fact that a number of causes of belt misalignment act simultaneously $[1,2,3,4]$. The most hidden causes are the belt camper and the uneven distribution of tension along the width of the traction core (manufacturing defect). Other reasons can be called man-made, depending on the technical culture of the staff. For example: the incorrect joining of belt segments; skew of segments in plan view or joining of segments (the width of the segments has tolerance \pm ) not along the axis, but along the conveyor-belt edge surface; installation of the linear flight with a deviation in plan view from a straight line or the installation of flight sections not perpendicularly to the sill (i.e., with the inclination of the flight cross-section); installation of roller supports, even on a straight line flight, not perpendicularly in plan view to the belt movement direction; installation of any drums with a skew in any plane; the increase in the resistance to rotation of the rollers (or failure) along one edge surface of the belt will lead to the redistribution of the tension along its width; the installation of the loading device that does not provide the symmetrical arrangement of the load on the receiving belt - all these errors will contribute to the belt misalignment.

\footnotetext{
"Corresponding author: yvm@kuzstu.ru
} 


\section{Materials and methods}

The belt misalignment can be prevented by minimizing the effect of the causes and by aligning the belt. Within the framework of the article, we will consider the aligning devices.

In practice, is the belt aligning by rotating the roller supports to a certain angle in plan view is used the most often [5] (Fig. 1, a) or by inclining in the vertical plane (Fig. 1, b). Simplicity wins. However, in order to avoid errors that are often encountered in practice, it should be noted that the force contributing to the belt misalignment is variable. It depends on the belt tension, i.e. from the amount of the load on the belt and from its varying position along the conveyor length. Therefore, belt aligning by rotating the roller supports to a certain angle in plan view (Fig. 1a) or inclining in the vertical plane (Figure 1, b) can lead to the opposite effect, to the misalignment in the opposite direction.
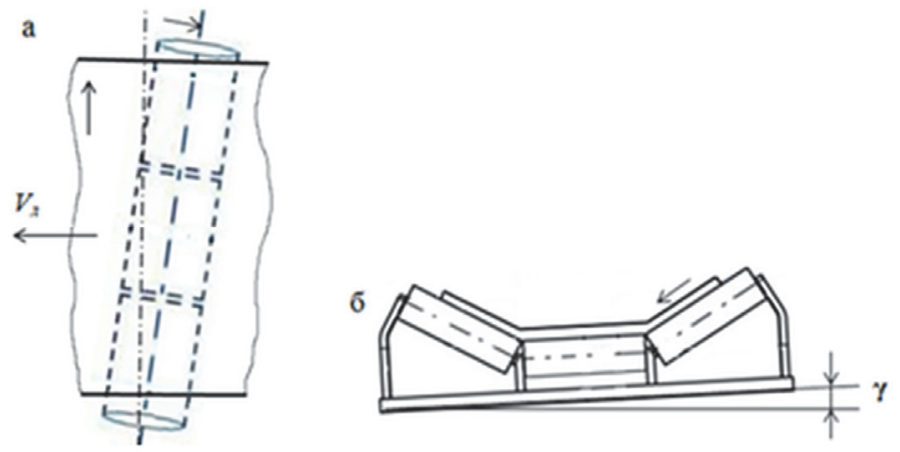

Fig.1. Ali gning the tape by rotating the support roller: a - in plan view, b - in the vertical plane.

In addition, the inclination of the roller supports at an angle $\gamma=4-5^{\circ}$ leads to the decrease in the receiving capacity by $10 \%$. The results are obtained by modeling the cross-section of the load on the belt; the inclination angle of the supporting roller being changed in vertical plane (Fig. 2).

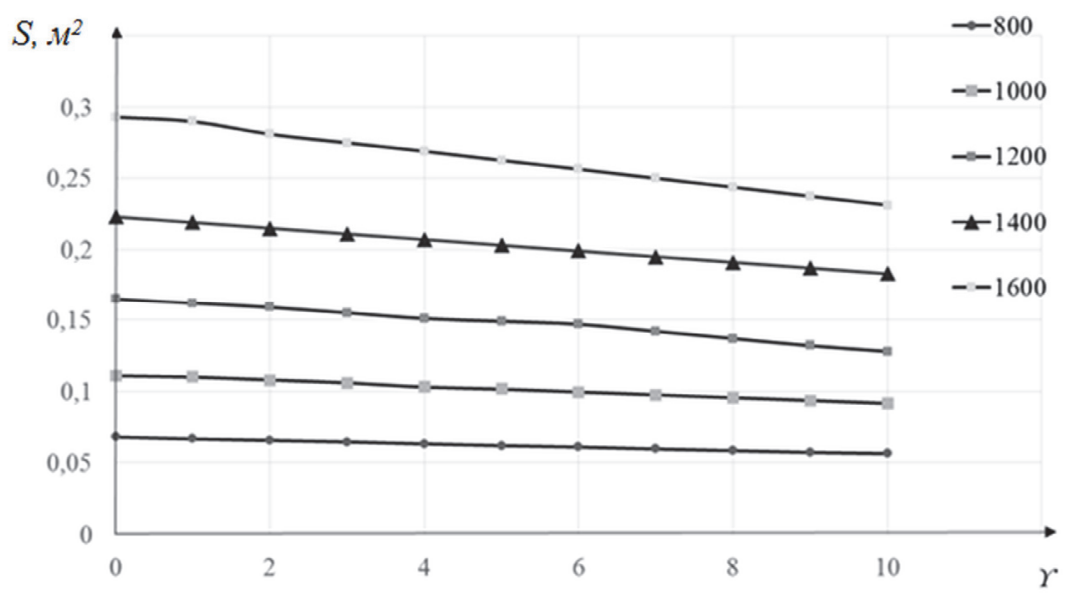

Fig. 2. Dependence of the sectional area of the load on the belt on the angle of inclination of the roller. 

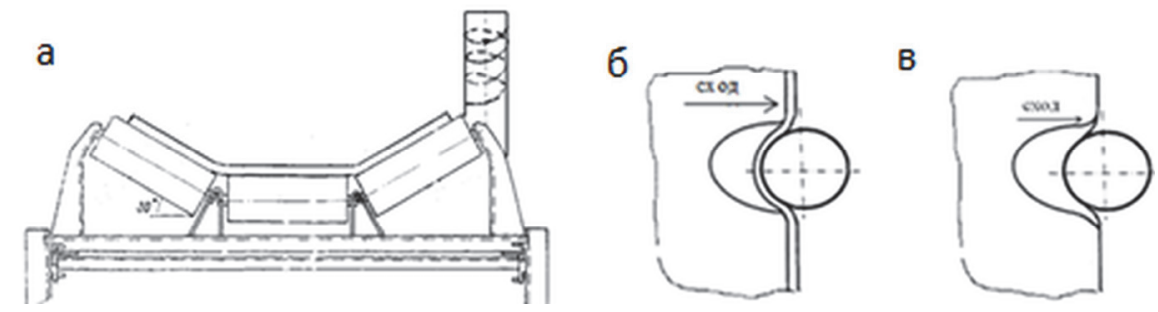

Fig. 3. Belt edge surface interaction with bump roller and deflector roller: a - bump roller, $\sigma$ - being pressed the belt edge surface move upwards, $\mathrm{B}-$ being pressed the belt edge surface move downward

Another common mistake is the use of bump rollers to counteract the belt misalignment (Fig. 3, a). Since the conveyor-belt edge surface rigidity is less than the roller shell rigidity, the belt will be pressed into the roller and can bend (move) upwards (Fig. 3, b). When moving upwards, the effect of "screwing" appears (Fig. 3, a), i.e. the belt moves up the bump roller up to the turning point. The process is accompanied by the spillage of the transported material. When pressed, the belt edge surface can be bent (shifted) downward relative to the bump roller (Fig. 3, c). In this case, the belt is directed into the space limited by the bump roller and fixed brackets of supporting rollers. This leads to increased wear of the belt edge surface by friction, up to the detachment of the pieces.

When the bump roller is installed incorrectly, i. e. the roller axis is inclined in the direction of the belt movement (or vice versa), the roller axis is not perpendicular to the plane of the belt interacting with it, and the processes described above are amplified. Stresses resulting from the interaction of the belt edge surface with the bump rollers, contribute to the intensive wear and the stratification of the side of the belt edge surface.

Self-aligning roller supports, which are rotational in plan view, which are represented by two types, are the most effective among the known means of belt aligning.

The first type is a self-aligning roller supports, rotatable in plan view, due to deflector roller levers (Fig. 4, a) [3]. To achieve the aligning effect, it is necessary that the lever arms and the gap between the belt edge surfaces and the deflector rollers allow the roller to rotate through the angle of $3-6^{\circ}$.
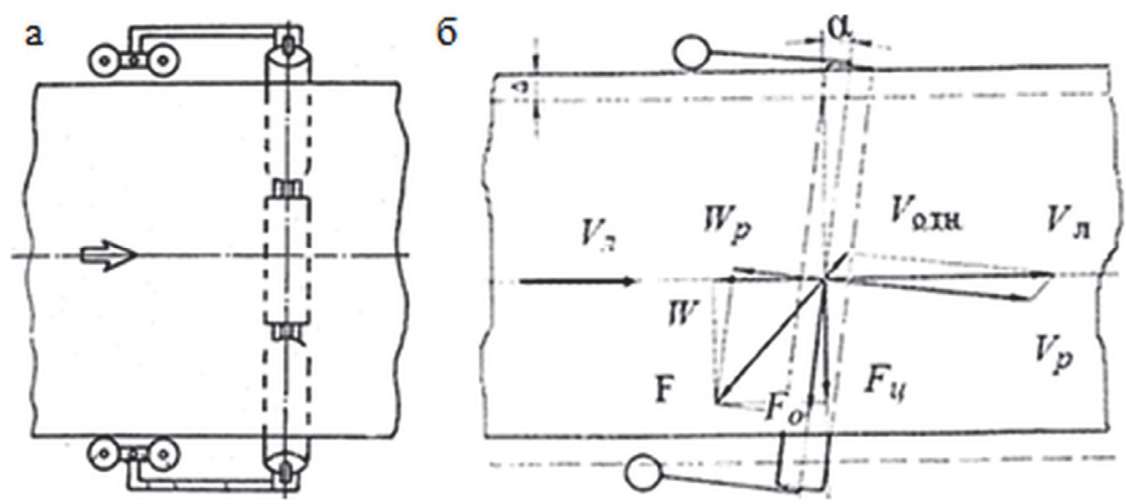

Fig. 4. Self-aligning roller support, rotational in plan view: a - with twin deflector rollers; $b$ - diagram of operating forces.

The advantage of such devices lies in the fact that the value of the aligning force varies depending on the value of the emerging force of the misalignment. When the belt moves along the roller support rotated by a certain angle, the vectors of their linear velocities $V_{p}$ and $V_{\Omega}$ are mismatched, as a result of which the relative velocity of the belt $V_{\text {отн }}$ (Fig. 4, b) 
appears. As a result, the friction force $F$ acts on the belt in the opposite direction. The projections of this force on the longitudinal axis which is perpendicular to the roller are its components: $W_{p}$ is the resistance to the belt movement; $F_{o}$ is the axial friction force. The projection of the axial friction force on the axis perpendicular to the longitudinal axis of the belt represents the aligning force opposing the misalignment of the tape.

The magnitude of the aligning force that occurs when turning the roller support in plan view depends on the force of the normal pressure on it from the side of the belt and the load and the clutch friction coefficient and the angle of rotation. For the effective operation of the self-aligning roller support, as well as the conveyor as a whole, it is necessary to ensure the groove of the belt (Fig. 5). That is, to fulfill the ratio

$$
0,5 B \leq(a+b+c) \leq B
$$

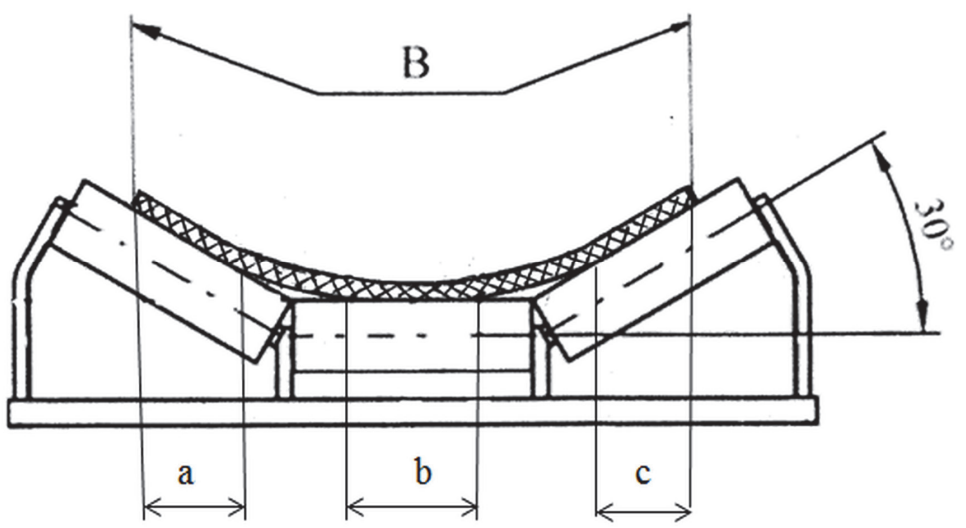

Fig. 5. The groove of the conveyer belt.

This is achieved by the fact that during the assembly of the conveyor the level of the surfaces of the tail and unloading drums should be at the level of the surface of the middle rollers of the supporting grooved rollers. The more contact, the more efficient will be the self-centering roller.

The disadvantage of such self-aligning roller supports is that the contact of the belt edge surfaces with the deflector roller, although with less effort compared to the bump rollers, occurs. And this contributes to the wear and the stratification of the side of the belt edge surface.
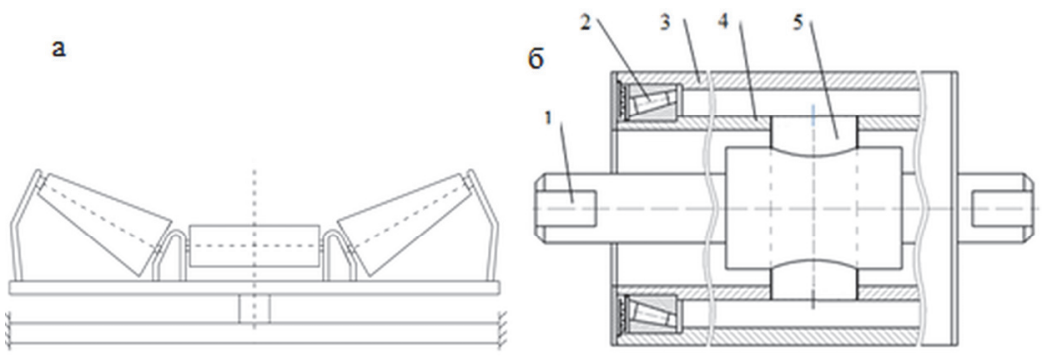

Fig. 6. Self-aligning roller support, rotational in plan view: a - with tapered side rollers (Tru-Trac Tapered Trough Tracker), $\sigma-$ with twin rotating shells (Tru-Trac Flat Return Tracker, Tru-Trac Trough Tracker).

\section{Conclusions}


The second type is the self-aligning roller support rotational in plan view by changing the position of the belt relative to the vertical turning node and creating greater force of interaction between the belt edge surface and the roller (Fig. 6, a) [6].

The most original solution is the self-aligning roller support with double rotational shells (Fig. 6, б) [7, 8]. The roller consists of an axis 1, in the central part of which there is a pin 5. The roller 4 is hinged on its inner shell. Tapered rollers 2 , on which the outer rotating shell 3 rests, are mounted at the ends of this shell. The radial clearance between the axis 1 and the inner shell 4 allows the roller to rotate relative to the pin 5 at the angle of 3 to $6^{\circ}$.

Advantages of self-aligning rollers of the firm "Tru-Trac":

- work with any direction of the belt movement,

- eliminate the wear and stratification of the belt edge surface.

Analysis of the examined devices shows that effective aligning of conveyor belts should be carried out only by self-aligning belt supports, rotational in plan view.

\section{References}

1. M. Noncic, Bulk Solids Handling, 32:1, 34 (2012)

2. F.L. Naples, Belt Conveyors for Bulk Materials, Sixth Edition published by Conveyor Equipment Manufacturers Association (Adelaida, CEMA, 2005).

3. R. Todd, L. J. Goldbeck, A. D. Marti, The Practical Resource for Total Dust \& Material Control, (Detroit, ASSG Found., 2002)

4. N. Schmidgall, Pit and Quarry, 95:12, 22 (2003)

5. F. Han, F. Xiong, R. He, Applied Mechanics and Materials, 66, 356 (2011)

6. G. Gerbert, Design Engineering Division 88, 443 (1996)

7. H. Cheng, S. Yanabe, Y. Iwata, H .Sato, T. Komatsuzaki, K. Sato, Transactions of the Japan Society of Mechanical Engineers, 68:10, 2911 (2002) 
